\title{
Performance of ion exchange resin as solid catalyst for the esterification of acetic acid with ethanol
}

\author{
B.A.N.N. Bamunusingha, E.C.L. De Silva and M.Y. Gunasekera* \\ Department of Chemical and Process Engineering, Faculty of Engineering, University of Moratuwa, Katubedda, Moratuwa.
}

Revised: 14 August 2015; Accepted: 23 September 2015

\begin{abstract}
Esterification of acetic acid with ethyl alcohol was carried out in the presence of a cation exchange resin. The resin used was Lewatit S1467, which is commercially available at a low cost and used commonly in the water treatment industry. The catalyst pretreated with $\mathrm{HCl}$ showed higher acetic acid conversions. The acidic cation exchange resin treated with $\mathrm{HCl}$ was examined for catalyst loading, reaction temperature and reusability. The highest acetic acid conversion of $58 \%$ was observed for acetic acid to ethanol mole ratio of $1: 1$ at $335 \mathrm{~K}$ with a $5.4 \%(\mathrm{w} / \mathrm{w})$ catalyst loading. The experimental data were fitted with the pseudo homogeneous model, and the heterogeneous models Langmuir-Hinshelwood (LH) and EleyRideal (ER). LH model provided a better agreement with the experimental kinetic data, and the activation energy was found to be about $58 \mathrm{kJmol}^{-1}$.
\end{abstract}

Keywords: Cation exchange resin, ethyl acetate, heterogeneous kinetics.

\section{INTRODUCTION}

The conventional way to produce esters is the esterification of carboxylic acids with alcohols catalysed by homogeneous catalysts such as sulphuric acid. These homogeneous catalyst reactions suffer from several drawbacks, such as the difficulties of catalyst separation from the reaction mixture, formation of side reactions and the corrosion of reactors (Peters et al., 2006). Therefore the attention of many research studies has turned to the replacement of homogeneous catalysts with solid acid catalysts. A variety of materials have been used as solid acid catalysts such as clays, zeolites and ion exchange resins in esterification reactions (Shanmugam et al., 2004). Although there exist many solid acid catalysts, cation-exchange resins are the most commonly used and have been proven to be effective in esterification reactions (Kirbaslar et al., 2001a; Liu \& Tan, 2001). Therefore esterification in the presence of ion exchange resins has been the recent subject of investigation by many researchers.

Kirbaslar et al. (2001a), have studied the esterification of acetic acid with ethyl alcohol catalysed by an acidic ionexchange resin, Amberlyst-15. They have investigated the effects of catalyst loading, reactant molar ratios, initial water concentration and temperature on the reaction rate. Similar studies have also been done by Toor et al. (2011) on the esterification of acetic acid with n-butanol and isobutanol over Amberlyst-15 catalyst, and by Akbay and Altiokka (2011) for the esterification reaction of acetic acid with n-amyl alcohol over Amberlyst-15, Amberlyst36 and Amberlite IR-120 catalysts. For the above reaction of acetic acid with n-amyl alcohol, the effect of external and internal mass transfer resistances on the overall rate have also been studied. The esterification of acetic acid with methyl alcohol catalysed by Amberlyst- 15 has been studied by Kirbaslar et al. (2001b). Their results have correlated with the homogeneous kinetic model and the activation energy was found to be $22.95 \mathrm{kJmol}^{-1}$.

Experiments for the esterification of n-amyl alcohol with propionic acid catalysed by three different cation exchange resins, namely, Amberlyst-15, Dowex 50W and Amberlite IR-120 were carried out within the temperature range of $333-348 \mathrm{~K}$ by Erdem and Cebe (2006). Similar to the study of Kirbaslar et al. (2001b), the kinetic data of this reaction have fitted well with the pseudo homogeneous kinetic model. Several studies have shown that the reaction kinetics of esterification fitted with Eley-Rideal (ER) kinetic model between adsorbed 
alcohol and unabsorbed carboxylic acid (Altiokka \& Citak, 2003; Liu et al., 2006; Izci et al., 2009). Due to the industrial importance of esterification over solid catalysts, there are a number of investigations reported in literature (Mazzotti et al., 1997; Lee et al., 2000; 2002; Lilja et al., 2002; Yadav \& Thathagar, 2002; Erdem \& Cebe, 2006; JagadeeshBabu et al., 2011; Ju et al., 2011; Tsai et al., 2011). However, studies on the esterification of acetic acid with ethanol over low cost ion exchange resins are lacking.

In this study, the esterification of acetic acid with ethyl alcohol catalysed by an inexpensive cation exchange resin available in the local market, namely Lewatit S1467, was investigated. This is a cation exchange resin of $\mathrm{Na}^{+}$form mainly used in the water treatment industry. The cost of this resin is much less compared to the commercially available strongacid cation exchange resin catalysts. In this study the influence of catalyst resin pre-treatment method, catalyst loading, reaction temperature and the reuse of catalyst on the conversion were analysed experimentally. The experimental kinetic data were correlated using models based on homogeneous and heterogeneous approaches.

\section{METHODS AND METERIALS}

\section{Materials and catalyst}

Acetic acid of $99.5 \%(\mathrm{w} / \mathrm{w})$ and ethanol of $99.5 \%(\mathrm{w} / \mathrm{w})$ purity were used as the reactants. The cation exchange resin, Lewatit S1467, which is available in the local market was used as the catalyst. The physical properties of this resin are shown in Table $1 . \mathrm{HCl}$ of $35 \%$ (w/w) was used for catalyst preparation. Before use, the ion exchange resin was soaked in $35 \%(w / w) ~ \mathrm{HCl}$ for $30 \mathrm{~min}$, rinsed several times with de-ionised water and dried in the oven at $363 \mathrm{~K}$ temperature to remove the moisture. The solvent used was $99 \%$ (w/w) n-heptane. Sodium hydroxide was used for the analysis. All the aqueous solutions used in the analysis were of analytical grade and prepared with distilled water.

\section{Procedure}

Acetic acid was charged into the reactor fitted with a total reflux condenser. The contents were heated to the desired temperature and kept constant using a temperature controlled water bath. A known amount of the catalyst was added to the contents in the reactor and mixed well using a magnetic stirrer. After the desired temperature was reached, ethanol preheated to the same temperature was quickly poured into the reactor. This moment was
Table 1: Physical properties of Lewatit S1467

\begin{tabular}{lc}
\hline Properties & Lewatit $\mathrm{S} 1467$ \\
\hline Physical form & Light brown spherical grain \\
Ionic form as shipped & $\mathrm{Na}^{+}$ \\
Moisture content $(\%)$ & $42-48$ \\
Grain size range $(\mathrm{mm})$ & $(0.05-0.6)>90 \%$ \\
Shipping density $(\mathrm{g} / \mathrm{L})$ & 820 \\
Maximum operating temperature $(\mathrm{K})$ & 393 \\
\hline
\end{tabular}

taken as the starting time of the reaction. In the case of initial reaction rate determination experiments, $n$-heptane was used as the solvent to prepare the solutions of desired reactant molar ratios. The required amount of one reactant and n-heptane were placed in the reactor. A known weight of the catalyst was added and heated to the desired temperature while stirring. The second reactant, which was preheated to the same temperature was then added. The total initial reactant volume used for all the experiments was $100 \mathrm{~mL}$. In all experimental runs, samples of $2 \mathrm{~mL}$ were withdrawn from the reactor at regular intervals and analysed to determine the unconverted acetic acid by titrating against $1 \mathrm{~N} \mathrm{NaOH}$ using phenolphthalein indicator.

\section{RESULTS AND DISCUSSION}

The cation exchange resin, Lewatit S1467 used in this study was in $\mathrm{Na}^{+}$form when it was commercially obtained from the local market. Initially the resin in its $\mathrm{Na}^{+}$form was used to catalyse the esterification reaction. Then the study was continued with the same resin after a pre-treatment to obtain the cation exchange resin of $\mathrm{H}^{+}$form. The effect of catalyst loading, reaction temperature and effect of reuse of the catalyst on acetic acid conversion with the pre-treated catalyst were investigated. Pseudo homogeneous and heterogeneous kinetic models, Langmuir-Hinshelwood (LH) and EleyRideal (ER) were applied to correlate the experimental kinetic data. Initial reaction rate variation was studied by changing one of the reactant concentrations, while keeping the others constant to analyse the consistency of heterogeneous model assumptions.

\section{Ion exchange resin in $\mathrm{Na}^{+}$form}

The effect of cation exchange resin Lewatit S1467 in the $\mathrm{Na}^{+}$form on the esterification reaction of acetic acid with ethyl alcohol was investigated. The resin was dried in the oven at $363 \mathrm{~K}$ for 4 hours. The experiment was carried out at the reaction temperature $335 \mathrm{~K}$ using $1.1 \%$ (w/w) dried resin in the reactant molar ratio of ethyl alcohol to acetic acid $1: 1$. The reaction mixture was agitated by 
a magnetic stirrer. The maximum catalyst performance after 5 hours showed a very low acetic acid conversion of about $3.5 \%$. This verifies the requirement of pretreatment of the resin used.

\section{Effect of catalyst pre-treatment method}

Use of strong acidic cation exchange resins of $\mathrm{H}^{+}$form as catalysts in the esterification reactions is commonly seen in literature (Kirbaslar et al., 2001a; Altiokka \& Citak, 2003). In this study, Lewatit S1467 resin, which is in $\mathrm{Na}^{+}$form was soaked in a $35 \%(\mathrm{w} / \mathrm{w}) \mathrm{HCl}$ solution for 30 minutes, washed with pure water and dried in the oven at $363 \mathrm{~K}$ to obtain the $\mathrm{H}^{+}$ionic form. The performance of resin samples of $\mathrm{H}^{+}$ionic form dried for 4 hours and 24 hours were studied. The results are shown in Figure 1. The results for the catalyst loading of $5.4 \%(\mathrm{w} / \mathrm{w})$ do not show a change in the acetic acid conversion while increasing the resin drying time from 4 to 24 hours. In order to verify this result another experiment was conducted to find out the influence of drying time on acetic acid conversion using $10.9 \%(\mathrm{w} / \mathrm{w})$ of catalyst at $335 \mathrm{~K}$ temperature with 1:1 molar ratio of reactants. The results are shown in Figure 1. It shows that catalyst drying time within the range of $4-24$ hours does not have a considerable effect on acetic acid conversion, and therefore a drying time of 4 hours was used in all experiments mentioned in the following sections.

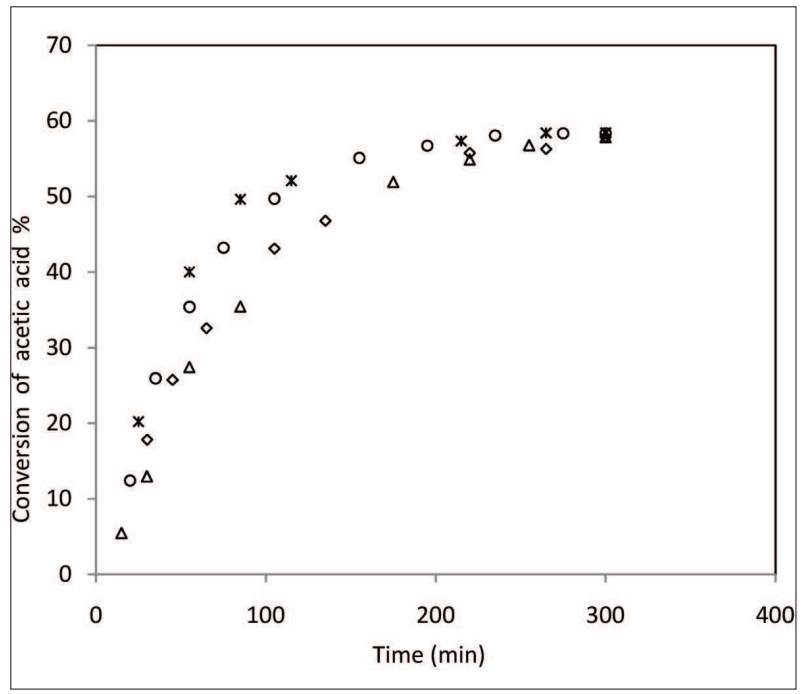

Figure 1: Conversion of acetic acid with time for reaction at $335 \mathrm{~K}$, molar ratio of ethyl alcohol to acetic acid 1:1, agitated by magnetic stirrer; for resin drying time 4 hours: catalyst loading 5.4 wt. $\%(\diamond)$ and 10.9 wt. \% $(\bigcirc)$ and for resin drying time 24 hours: catalyst loading 5.4 wt. $\%(\Delta)$ and 10.9 wt. $\%(*)$.

\section{Effect of catalyst loading}

Previous studies have reported that it was not practical to use more than $10 \%(\mathrm{w} / \mathrm{w})$ catalyst loading for heterogeneously catalysed reactions (Alqahtani et al., 2005). In this study, catalyst loadings of $1.1 \%, 5.4 \%$ and $10.9 \%(\mathrm{w} / \mathrm{w})$ in $100 \mathrm{~mL}$ of reaction mixture were analysed and the results are illustrated in Figure 2. An increase in the catalyst loading has led to an increase of reaction rate. This is due to an increase in active catalytic sites available for the reaction as the catalyst load increases. Several other researchers also have reported that the increase of catalyst loading has caused the increase of reaction rate in the esterification of carboxylic acid with alcohol over cation exchange resins (Erdem \& Cebe, 2006; Delgado et al., 2007; Izci \& Hosgun, 2007). An equilibrium conversion is observed for catalyst loadings of $10.9 \%$ and $5.4 \%(\mathrm{w} / \mathrm{w})$ after about 5 hours, although for the loading of $1.1 \%(\mathrm{w} / \mathrm{w})$ an equilibrium conversion of acetic acid was not reached at this time. The equilibrium conversions for catalyst loadings $5.4 \%$ and $10.9 \%(\mathrm{w} / \mathrm{w})$ do not show a significant difference. This indicates that the equilibrium conversion is independent of the catalyst loading. The conversion rate is also similar in $5.4 \%$ and $10.9 \%(\mathrm{w} / \mathrm{w})$ and higher compared to that of $1.1 \%(\mathrm{w} / \mathrm{w})$. This agrees well with what Toor et al. (2011) have mentioned, that as at higher catalyst loadings the rate of mass transfer is high and therefore there is no significant increase in the rate although the load is increased further.

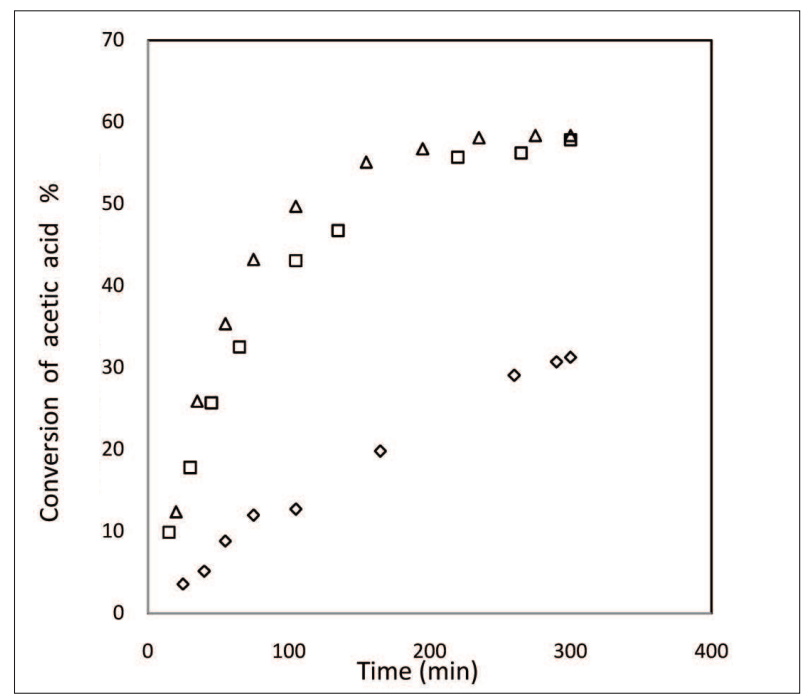

Figure 2: Conversion of acetic acid with time for different catalyst loadings: $1.1 \%(\mathrm{w} / \mathrm{w})(\diamond) ; 5.4 \%$ (w/w) $(\square) ; 10.9 \%$ $(\mathrm{w} / \mathrm{w})(\Delta)$. (reaction at $335 \mathrm{~K}$, molar ratio of ethyl alcohol to acetic acid $1: 1$, agitated by magnetic stirrer). 


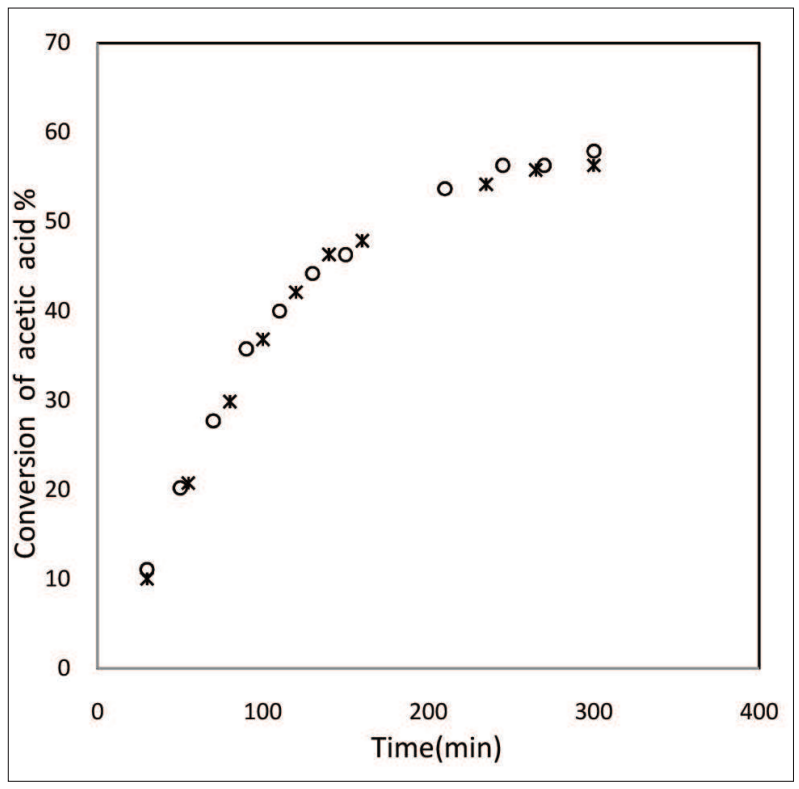

Figure 3: Conversion of acetic acid with time over fresh catalyst: run $1(\bigcirc)$ and reused catalyst: run $2(*)$ at $335 \mathrm{~K}$, molar ratio of ethyl alcohol to acetic acid 1:1 agitated by magnetic stirrer, catalyst loading $5.4 \%(\mathrm{w} / \mathrm{w})$.

\section{Reuse of catalyst}

Reuse of the catalyst is an important aspect in industrial reactions. It is useful to have an environmentally friendly process while being economical. For this study, once used resin from a reaction run with a loading of $5.4 \%$ $(\mathrm{w} / \mathrm{w})$ catalyst was recovered by filtration. The recovered resin was washed with pure water, dried at $363 \mathrm{~K}$ and the weight was adjusted to have a total weight of $5.4 \%(\mathrm{w} / \mathrm{w})$ by adding makeup pre-treated resin. The esterification reaction experiment was carried out using this resin sample as previously with fresh reactants. The acetic acid conversion results are shown in Figure 3 for the reaction with fresh catalyst (run 1) and for the same with reused catalyst (run 2). As seen in Figure 3 after the reuse of catalyst the conversion has slightly decreased from $58 \%$ to $56 \%$ after 5 hours indicating a good ability to reuse the catalyst. A similar decrease in the conversion has been observed by other researchers. According to Shanmugam et al. (2004), the conversion of methacrylic acid has shown a decrease from $83 \%$ to $73 \%$ after three reuse cycles in the esterification of triethylene glycol with methacrylic acid catalysed by a heteropoly solid acid catalyst.

\section{Effect of reaction temperature}

Temperature is one of the parameters, which influences the rate of reaction. In this study, the effect of temperature

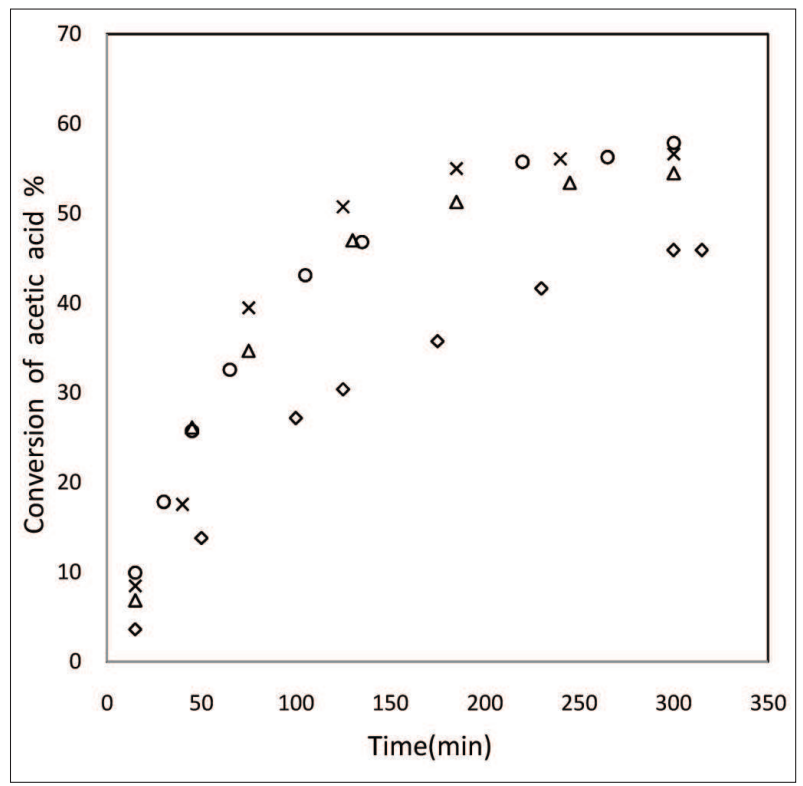

Figure 4: Conversion of acetic acid with time for different reaction temperatures: $325 \mathrm{~K}(\diamond) ; 335 \mathrm{~K}(\bigcirc) ; 345 \mathrm{~K}(\Delta)$ and $355 \mathrm{~K}$ $(\times)$; molar ratio of ethyl alcohol to acetic acid 1:1, agitated by magnetic stirrer, catalyst loading $5.4 \%(\mathrm{w} / \mathrm{w})$.

on the esterification reaction was studied over a temperature range from 325 to $355 \mathrm{~K}$ using Lewatit $\mathrm{S} 1467$ as the catalyst pre-treated with $\mathrm{HCl}$ as described previously and dried for 4 hours. The results (Figure 4) show that the reaction rate increases with the increase in temperature from 325 to $335 \mathrm{~K}$. A similar study carried out by Kirbaslar et al. (2001a), for the esterification of acetic acid with ethanol over Amberlyst-15 has also shown an increase in the conversion of acetic acid when increasing the temperature from $323 \mathrm{~K}$ to $353 \mathrm{~K}$. Similar trends have been observed in many studies as well (Kirbaslar et al., 2001b; Altiokka \& Citak, 2003; Erdem \& Cebe, 2006; Delgado et al., 2007; Izci \& Hosgun, 2007; Toor et al., 2011). After five hours it was observed that an equilibrium conversion has been achieved in the temperature range $335 \mathrm{~K}-355 \mathrm{~K}$. The equilibrium conversion in this range was nearly equal. The above results show that a $58 \%$ acetic acid conversion can be achieved in the esterification of equi molar ratio of reactants at $335 \mathrm{~K}$ reaction temperature over $5.4 \%(\mathrm{w} / \mathrm{w})$ of Lewatit S1467 catalyst soaked in $35 \%(\mathrm{w} / \mathrm{w}) \mathrm{HCl}$ for 30 minutes and dried at $363 \mathrm{~K}$ for 4 hours. The information on reaction rate and acetic acid conversion at different temperatures is useful for the calculation of activation energy for this reaction, and also useful in deriving the function relating the rate constant with the temperature. 


\section{Mass transfer resistance}

Although kinetic experiments were done assuming that the influence of mass transfer resistances was negligible, it is important to identify the reaction rate controlling parameters. The overall reaction rate of a heterogeneous reaction not only depends upon the kinetics of the reaction, but also on the internal and external diffusion rates and adsorption and desorption rates of the reactants and products. Each of these activities of reactants or product species becomes an element of the series of steps involved in the total heterogeneous reaction. The slowest step of this series becomes the rate limiting step (Fogler, 2006). Therefore it is essential to evaluate the mass transfer resistances prior to the study of the reaction kinetics.

The work of Chakrabarti and Sharma (1993) has established that external diffusion does not control the overall reaction rate unless the agitation speed is very low or the viscosity of reactant mixture is very high. The studies done by Alqahtani et al. (2005), and Delgado et al. (2007), have also reported that when the agitation speed is changed from a speed above a certain level to higher speeds, the rate of reaction doesn't change significantly for a similar kind of reaction setup as used in this study with ion exchange resin catalyst. Based on these observations, the agitation in this research was maintained at a constant level in each run assuming that the external mass transfer resistance under this condition was negligible.

The study done by Zhang et al. (2004), has used a theoretical procedure to prove that the internal mass transfer effects are negligible. Their analysis is based on the Thieles' modulus, which is also of interest in this study. Thieles' modulus for a spherical particle involved in a first order reaction can be given by equation (1) (Smith, 1970).

$\varphi_{s}=\frac{r_{s}}{3} \sqrt{\frac{k_{1} \rho_{p}}{D_{e}}}$

Thieles' modulus is denoted by $\varphi_{s}$ and, $r_{s}$ and $k_{1}$ represent the mean radius of spherical particles and the forward rate constant, respectively. Effective diffusion coefficient is given by $D_{e}$ and, $\rho_{p}$ is the density of solid catalyst particle. Further, the ratio between the actual reaction rate for the whole pellet to the rate evaluated at outer surface conditions is identified as the effectiveness factor, which tends to reach unity as the mass transfer resistances are reduced. Using the relationship curves of Thieles' modulus and the effectiveness factor (Smith, 1970), it can be observed that the effectiveness factor is closer to unity when $\varphi_{s} \leq 1 / 3$ making inequality (2) to be a condition to neglect the internal mass transfer resistances.

$r_{s}^{2} \frac{k_{1} \rho_{p}}{D_{e}} \leq 1$

The elimination of $k_{1}$ from equation (2) using the simple first order kinetic equation yields equation (3) as the condition to check the significance of internal resistances. However since the relationship curves of the effectiveness factor with the Thieles' modulus for different reaction orders overlap for $\varphi_{s} \leq 1 / 3$, inequality (3) is applicable for higher orders as well (Smith, 1970).

$r_{s}^{2} \frac{r_{p} \rho_{p}}{C_{s} D_{e}} \leq 1$

In inequality (3), $C_{s}$ represents the concentration of the reference species in the reaction medium. The effective diffusion coefficient was defined as equation (4) (Fogler, 2006).

$D_{e}=\frac{D_{A} \varepsilon}{l}$

Where $D_{A}$ is the molecular diffusion coefficient, $\tau$ and $\varepsilon$ are tortuosity and porosity of the catalyst particle, respectively. The typical values for $\tau / \varepsilon$ are between 0.25 and 0.5 for most of the resin catalysts (Zhang et al., 2004). The value 0.5 was taken as the ratio $\tau / \varepsilon$ for the calculations in this study. Furthermore, $D_{A}$ was obtained using the Wilke-Chang equation [equation (5)] (Reid et al., 1987).

$D_{A}=\frac{7.4 \times 10^{-8} \sqrt{\varnothing^{\prime} M T}}{\left(V_{A}\right)^{0.6} \mu}$

Association factor, temperature and molar mass of the solvent are represented by $\varnothing^{\prime}, T$ and $M$, respectively in equation (5). $V_{A}$ is the molar volume at boiling point and $\mu$ is the viscosity of the solution. For solvent mixtures, the relationship $\sqrt{\varnothing^{\prime} M}=\sqrt{\sum x_{i} \varnothing_{i}^{\prime} M_{i}}$ can be used to calculate the associated molar mass. In order to consider the most critical situation in inequality (3), the minimum effective diffusion coefficient value has to be considered. According to equations (4) and (5), effective diffusion coefficient $\left(D_{e}\right)$ reduces with the increase of $V_{A}$ and $\mu$. Therefore using the highest observable values for $V_{A}$ and $\mu$, 
the minimum expectable value for diffusion coefficient $\left(D_{e}\right)$ can be calculated. In this study $D_{e}$ was found to be equal to $9.9 \times 10^{-10} \mathrm{~m}^{2} \mathrm{~s}^{-1}$. Left hand side of the inequality (3) can be calculated using the initial reactant concentrations and measured reaction rate. The calculated value was 0.46 , which is less than unity, obeying the inequality. This indicates that internal mass transfer resistances can be neglected at the temperatures and combinations of the species met at the experimental runs in this study.

\section{Kinetic modelling}

The kinetics of the esterification reaction catalysed by acidic cation exchange resin has been discussed under both homogeneous and heterogeneous approaches. The simple pseudo homogeneous second order model is applicable to many ion exchange resin catalysed reactions. Here, complete swelling of the resin catalyst is assumed and therefore easy access of the reactants to the active sites. However this model does not take into account the sorption of reactants onto the catalyst surface. More complex heterogeneous models such as Langmuir-Hinshelwood (LH) and Eley-Rideal (ER) consider sorption effects of reactants in their mechanisms. In the LH model, before the chemical reaction takes place, it is assumed that all the reactants are adsorbed on the catalyst surface whereas in the ER model the reaction is assumed to take place between an adsorbed and a non-adsorbed reactant. Therefore, the pseudo homogeneous model and the heterogeneous models LH and ER were applied for correlating the kinetic data at different temperatures.

\section{Homogeneous kinetic study}

The pseudo homogeneous second order model applied in the ion exchange resin catalysed reactions assumes that the reaction media is highly polar and that there is complete swelling of the resin matrix (Helfferich, 1962). The integrated form of the pseudo homogeneous kinetic model is given by equation (6) (Altiokka \& Citak, 2003). It was assumed that the concentrations of species are in agreement with the stoichiometry of the reaction.

$\ln \left[\frac{X_{A e}-\left(2 X_{A e}-1\right)-X_{A}}{X_{A e}-X_{A}}\right]=2 \cdot k 1\left(\frac{1}{X_{A e}}-1\right) C_{A 0} \cdot t$

Where,

$t \quad$ Time

$C_{A 0} \quad$ Initial concentration of acetic acid, $\mathrm{mol} \mathrm{L}^{-1}$

$X_{A} \quad$ Conversion of acetic acid at time $\mathrm{t}$

$X_{A e} \quad$ Conversion of acetic acid at equilibrium

$k 1 \quad$ Forward rate constant, $\mathrm{L} \mathrm{mol}^{-1} \mathrm{~min}^{-1}$
The activation energy can be calculated using the Arrhenius equation, which is written in the logarithmic form as given in equation (7):

$\ln k 1=\ln K-\frac{E_{a}}{R T}$

Where,

k1 Forward rate constant, $\mathrm{L} \mathrm{mol}^{-1} \mathrm{~min}^{-1}$

$K \quad$ Frequency of collisions

$E_{a} \quad$ Activation energy, $\mathrm{kJ} \mathrm{mol}^{-1}$

$T \quad$ Temperature, $\mathrm{K}$

$R \quad$ Gas constant, $\mathrm{kJ} \mathrm{mol}^{-1} \mathrm{~K}^{-1}$

The experimental data observed in Figure 4 were used to plot the left hand side of equation (6) versus time as shown in Figure 5. The forward rate constant at specified temperatures can be calculated using the slope of the straight line passing through the origin. These forward rate constant data were then plotted on a $(\ln k 1)$ against $(1 / T)$ graph as shown in Figure 6. The straight line fitting these data as indicated in equation (7) gives a coefficient of determination $\left(\mathrm{R}^{2}\right)$ of $81.26 \%$.

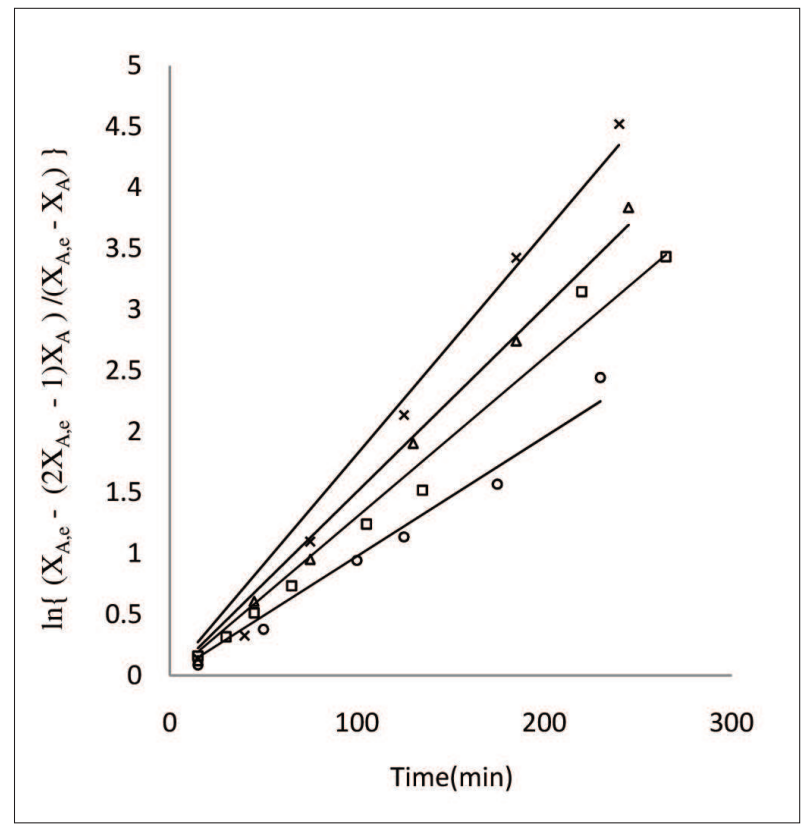

Figure 5: LHS of equation (6) vs. time at different temperatures: $325 \mathrm{~K},(\bigcirc) ; 335 \mathrm{~K},(\square) ; 345 \mathrm{~K},(\Delta) ; 355 \mathrm{~K},(\times)$; agitated by magnetic stirrer, catalyst loading $5.4 \%(\mathrm{w} / \mathrm{w})$, molar ratio of ethyl alcohol to acetic $1: 1$. 


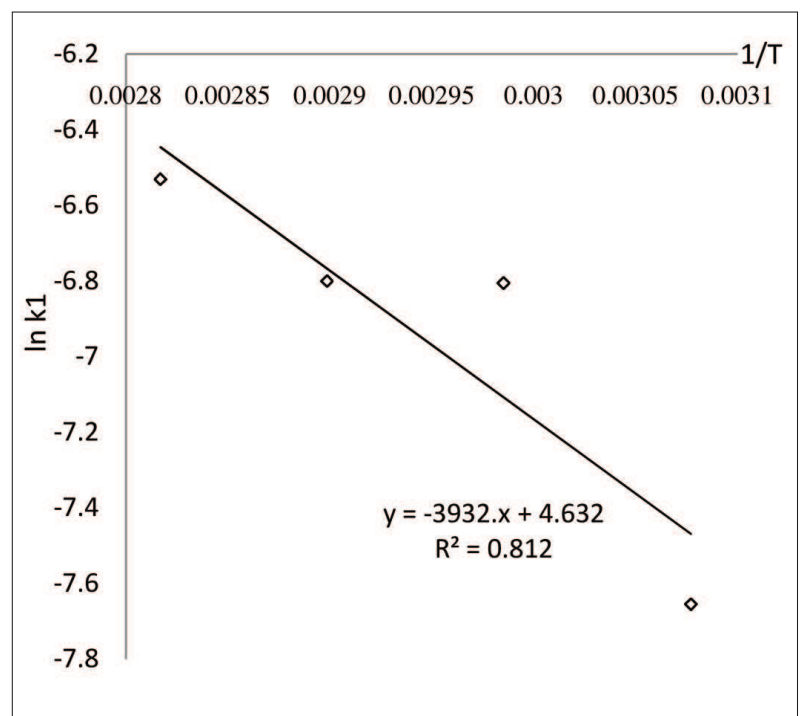

Figure 6: Arrhenius plot of forward rate constant for the esterification reaction using homogeneous reaction kinetics.

\section{Heterogeneous kinetic study}

The heterogeneous models Langmuir-Hinshelwood (LH) and Eley-Rideal (ER) were investigated to represent the reaction kinetics. A detailed description of the modelling of heterogeneous acid catalysed reactions is available in Carberry (1976). Both these models have been derived from the basic kinetic expression given by equation (8) under homogeneous conditions (Schwarzer, 2006),

$r=k\left(C_{A c} C_{E}-\frac{C_{E A} C_{W}}{K_{e q}}\right)$

In equation (8), $r$ and $k$ denote the reaction rate and the forward rate constant with respective to acetic acid, respectively. Notation $C$ represents the concentrations with subscripts $A c, E, E A$ and $W$ representing acetic, ethanol, ethyl acetate and water, respectively. LangmuirHinshelwood model applied for the esterification reaction accounts for the adsorption of both reactants, acetic and ethanol followed by the desorption of products, ethyl acetate and water. Therefore equation (8) can be written using fractional coverage terms as in equation (9), where fractional coverage $(\theta)$ is identified as the fraction of the sites occupied by a particular species from the total number of sites on the catalyst surface (Schwarzer, 2006).

$r=k\left(\theta_{A c} \theta_{E}-\frac{\theta_{E A} \theta_{W}}{K_{e q}}\right)$
The use of Langmuir isotherms for each species to eliminate the fractional coverage terms in equation (9) yields the Langmuir-Hinshelwood model equation as shown in equation (10) (Schwarzer, 2006).

$r=k \frac{C_{A c} C_{E}-\frac{C_{E A} C_{W}}{K_{e q}}}{\left(1+k_{w} C_{W}+k_{E} C_{E}+k_{A c} C_{A c}+k_{E A} C_{E A}\right)^{2}}$

where,

$k_{i}=\frac{c_{i-s}}{C_{i} c_{s}}, \quad K_{e q}=\left(\frac{C_{E A} \times C_{W}}{C_{A c} \times C_{E}}\right)_{e q}$

In equation (10), $k$ represents the reaction constant and $k_{i}$ represents the adsorption coefficients where $i$ may be water, ethanol, acetic acid or ethyl acetate. The notations $c_{s}$ and $c_{i-s}$ denote the concentration of vacant cites on the catalyst surface, and the concentration of component $i$ at the catalyst surface, respectively. However all four types of species in the reaction mixture compete with each other to adsorb onto a particular site of the resin particle. The studies of Delgado et al. (2007), and Zhang et al. (2004), show that water and ethanol have much stronger adsorption into a cation exchange resin during the esterification reaction of lactic acid with ethanol. The same was assumed in this study and therefore the adsorption of acetic acid and ethyl acetate are neglected from equation (10). This modifies the LH model equation as in equation (11), facilitating less complexity in parameter estimation.

$r=k \frac{C_{A c} C_{E}-\frac{C_{E A} C_{W}}{K_{e q}}}{\left(1+k_{w} C_{W}+k_{E} C_{E}\right)^{2}}$

The adsorption of only one reactant is considered in the ER model, while the other reactant is within the reaction medium during the reaction. Therefore in this case, equation (8) can be written as equation (12).

$r=k\left(C_{A c} \theta_{E}-\frac{C_{E A} \theta_{W}}{K_{e q}}\right)$

Fractional coverage terms approximated using the relevant Langmuir adsorption isotherms yields equation (13) as the Eley-Rideal model equation with similar notations as in equation (10) (Schwarzer, 2006). 
$r=k \frac{C_{A c} C_{E}-\frac{C_{E A} C_{W}}{K_{e q}}}{\left(1+k_{w} C_{W}+k_{E} C_{E}\right)}$

The results of experimental runs under the temperatures $325,335,345$ and $355 \mathrm{~K}$ were correlated simultaneously by using the LH and ER kinetic models. In addition to equations (11) and (13) the reaction rate can also be expressed using the rate of decomposition as,

$r=\frac{c_{A c, 0}}{W}\left(\frac{d X}{d t}\right)$

Notations, $C_{A c, 0}$ and $W$ represent the initial acetic acid concentration and catalyst loading respectively, while $r$ being the reaction rate with respect to acetic acid. Here $X$ and $t$ are the fractional decomposition and time (Fogler, 2006). Equation (14) together with equations (11) and (13) provides the relationships between decomposition and time.

The estimation of parameters $k, k_{W}$ and $k_{E}$ was done by minimising the sum of residual squares (SRS) of fractional decompositions at experimental kinetic data points (equation 15). Subscripts 'exp' and 'cal' means experimental and calculated values of fractional decompositions, respectively. Minimisation of the equation (15) was done using a user-built programme in Matlab software package. Integration of the relationship of decomposition $(X)$ and time $(t)$ was done numerically with the fourth order Runge-Kutta method. Furthermore, the mean relative deviation (MRD) shown in equation (16) was determined for the sets of parameters, which best fit each of the evaluated models in order to facilitate convenient comparison among these models. A lower $M R D$ value indicates a better data fit to the model. In equation (16), $N$ is the number of kinetic data observation points and $X$ is the fractional decomposition with subscripts 'calc' and 'exp' being its' calculated and experimental values.

$S R S=\sum_{N}\left(X_{\text {exp }}-X_{\text {calc }}\right)^{2}$

$M R D=\frac{1}{N}\left(\sum_{N}\left|\frac{X_{\text {calc }}-X_{\text {exp }}}{X_{\text {exp }}}\right|\right) \times 100 \%$
A summary of the results of parameters estimated is given in Table 2. Considering the results of all four temperatures, it is observed that the LH model correlates experimental results better than the ER model. Further, out of the four temperatures studied, the kinetic data at $335 \mathrm{~K}$ are obeying the ER and LH models with the lowest MRD \%. The plot of the modelled decomposition curve for LH model, and its experimental decomposition values at $335 \mathrm{~K}$ are illustrated in Figure 7. Rate constants determined from the LH model were put in an Arrhenius plot and are shown in Figure 8. Comparison of the coefficient of determination $\left(\mathrm{R}^{2}\right)$ of the Arrhenius plots of the pseudo homogeneous model (Figure 6) and the LH model (Figure 8) shows that kinetic data fit the LH model better than the homogeneous model. The activation

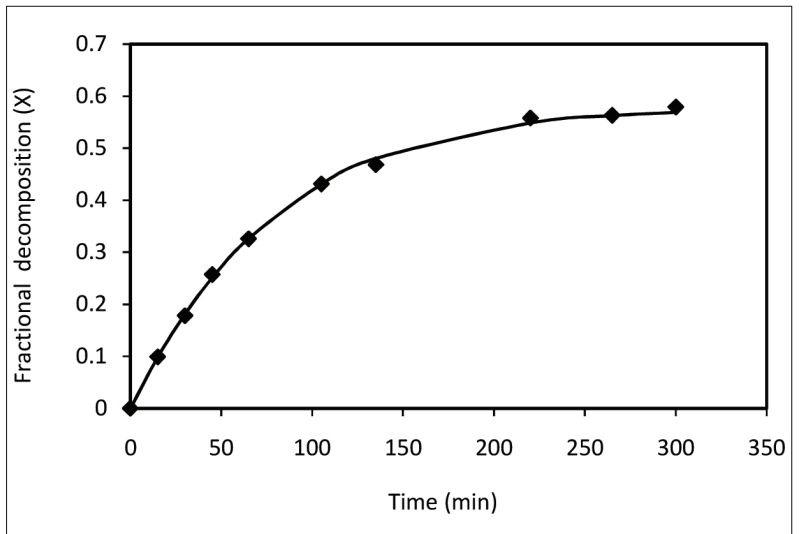

Figure 7: $\quad$ Conversion $v$ s time at $335 \mathrm{~K}$, acetic: ethanol $=1: 1$, catalyst loading $=50 \mathrm{gL}^{-1},(\diamond)$ experimental value, $(-)$ results of the LH model.

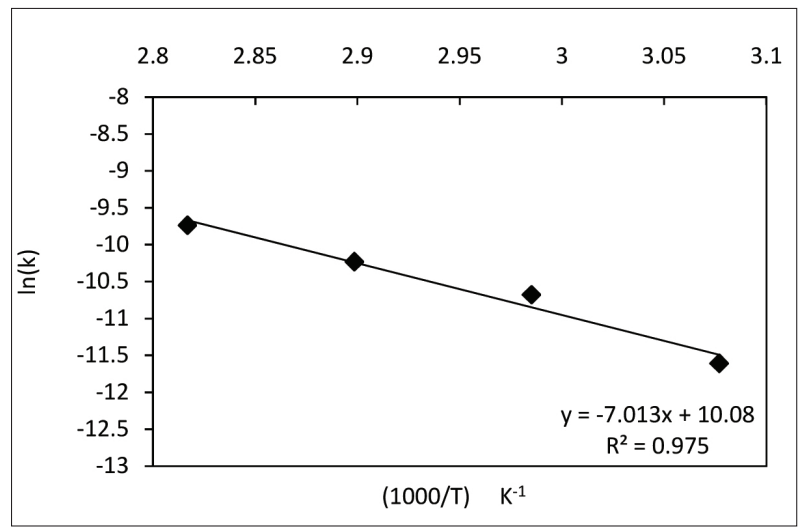

Figure 8: Arrhenius plot for rate constants calculated using LH model. 
Table 2: Summary of reaction parameters

\begin{tabular}{|c|c|c|c|}
\hline Temperature & Parameters $^{a}$ & Value & $\operatorname{MRD}(\%)$ \\
\hline \multirow[t]{8}{*}{$325 \mathrm{~K}$} & LH model & & \\
\hline & $\mathrm{k}\left(\mathrm{L}^{2} \mathrm{~g}^{-1} \mathrm{~mol}^{-1} \mathrm{~min}^{-1}\right)$ & $9.1 \times 10^{-6}$ & $5.53 \%$ \\
\hline & $\mathrm{k}_{\mathrm{W}}\left(\mathrm{L} \mathrm{mol}^{-1}\right)$ & $1.8 \times 10^{-2}$ & \\
\hline & $\mathrm{k}_{\mathrm{E}}\left(\mathrm{L} \mathrm{mol}^{-1}\right)$ & $5.5 \times 10^{-2}$ & \\
\hline & ER model & & \\
\hline & $\mathrm{k}\left(\mathrm{L}^{2} \mathrm{~g}^{-1} \mathrm{~mol}^{-1} \mathrm{~min}^{-1}\right)$ & $3.2 \times 10^{-5}$ & $6.76 \%$ \\
\hline & $\mathrm{k}_{\mathrm{W}}\left(\mathrm{L} \mathrm{mol}^{-1}\right)$ & 0 & \\
\hline & $\mathrm{k}_{\mathrm{E}}\left(\mathrm{L} \mathrm{mol}^{-1}\right)$ & $8.7 \times 10^{-2}$ & \\
\hline \multirow[t]{8}{*}{$335 \mathrm{~K}$} & LH model & & \\
\hline & $\mathrm{k}\left(\mathrm{L}^{2} \mathrm{~g}^{-1} \mathrm{~mol}^{-1} \mathrm{~min}^{-1}\right)$ & $2.3 \times 10^{-5}$ & $1.28 \%$ \\
\hline & $\mathrm{k}_{\mathrm{W}}\left(\mathrm{L} \mathrm{mol}^{-1}\right)$ & $4.3 \times 10^{-2}$ & \\
\hline & $\mathrm{k}_{\mathrm{E}}\left(\mathrm{L} \mathrm{mol}^{-1}\right)$ & $5.7 \times 10^{-2}$ & \\
\hline & ER model & & \\
\hline & $\mathrm{k}\left(\mathrm{L}^{2} \mathrm{~g}^{-1} \mathrm{~mol}^{-1} \mathrm{~min}^{-1}\right)$ & $7.7 \times 10^{-5}$ & $1.26 \%$ \\
\hline & $\mathrm{k}_{\mathrm{W}}\left(\mathrm{L} \mathrm{mol}^{-1}\right)$ & $5 \times 10^{-2}$ & \\
\hline & $\mathrm{k}_{\mathrm{E}}\left(\mathrm{L} \mathrm{mol}^{-1}\right)$ & $9.4 \times 10^{-2}$ & \\
\hline \multirow[t]{8}{*}{$345 \mathrm{~K}$} & LH model & & \\
\hline & $\mathrm{k}\left(\mathrm{L}^{2} \mathrm{~g}^{-1} \mathrm{~mol}^{-1} \mathrm{~min}^{-1}\right)$ & $3.6 \times 10^{-5}$ & $6.33 \%$ \\
\hline & $\mathrm{k}_{\mathrm{W}}\left(\mathrm{L} \mathrm{mol}^{-1}\right)$ & $4 \times 10^{-2}$ & \\
\hline & $\mathrm{k}_{\mathrm{E}}\left(\mathrm{L} \mathrm{mol}^{-1}\right)$ & $7.5 \times 10^{-2}$ & \\
\hline & ER model & & \\
\hline & $\mathrm{k}\left(\mathrm{L}^{2} \mathrm{~g}^{-1} \mathrm{~mol}^{-1} \mathrm{~min}^{-1}\right)$ & $9.9 \times 10^{-5}$ & $6.62 \%$ \\
\hline & $\mathrm{k}_{\mathrm{W}}\left(\mathrm{L} \mathrm{mol}^{-1}\right)$ & $3 \times 10^{-2}$ & \\
\hline & $\mathrm{k}_{\mathrm{E}}\left(\mathrm{L} \mathrm{mol}^{-1}\right)$ & $1.3 \times 10^{-1}$ & \\
\hline \multirow[t]{8}{*}{$355 \mathrm{~K}$} & LH model & & \\
\hline & $\mathrm{k}\left(\mathrm{L}^{2} \mathrm{~g}^{-1} \mathrm{~mol}^{-1} \mathrm{~min}^{-1}\right)$ & $5.9 \times 10^{-5}$ & $9.95 \%$ \\
\hline & $\mathrm{k}_{\mathrm{W}}\left(\mathrm{L} \mathrm{mol}^{-1}\right)$ & $6.4 \times 10^{-2}$ & \\
\hline & $\mathrm{k}_{\mathrm{E}}\left(\mathrm{L} \mathrm{mol}^{-1}\right)$ & $9.3 \times 10^{-2}$ & \\
\hline & ER model & & \\
\hline & $\mathrm{k}\left(\mathrm{L}^{2} \mathrm{~g}^{-1} \mathrm{~mol}^{-1} \mathrm{~min}^{-1}\right)$ & $1.9 \times 10^{-4}$ & $9.99 \%$ \\
\hline & $\mathrm{k}_{\mathrm{W}}\left(\mathrm{L} \mathrm{mol}^{-1}\right)$ & $1 \times 10^{-1}$ & \\
\hline & $\mathrm{k}_{\mathrm{E}}\left(\mathrm{L} \mathrm{mol}^{-1}\right)$ & $2.4 \times 10^{-1}$ & \\
\hline
\end{tabular}

energy according to the LH mechanism was calculated to be $58.29 \mathrm{kJmol}^{-1}$. Hangx et al. (2001), have observed an activation energy value of $48.3 \mathrm{~kJ} \mathrm{~mol}^{-1}$ in their study on ethyl acetate production catalysed by Purolite CT179. This shows that the acidic cation exchange resin, Lewatit S1467 has a higher activation energy compared to that of the strong cation exchange resin Purolite 179. This high value of the activation energy indicates that the reaction is kinetically controlled.

\section{Heterogeneous experimental data and rate mechanism}

The rate mechanism was analysed using experimental observations from the heterogeneous catalytic reaction. The experiments were carried out by changing the concentration of one of the reactants while keeping the other parameters constant. For each of these runs the initial reaction rate was determined. The results of initial rate of disappearance of acetic acid for various initial concentrations of acetic acid and water are illustrated in Figures 9 and 10, respectively.

As seen in Figure 9, the initial reaction rate increases linearly with the acid concentration. This shows that the reaction is first order with respect to acetic acid, and the same has been considered in the heterogeneous reaction mechanisms shown in ER and LH model equations. Here the adsorption of acetic acid onto the catalyst surface is not evident. In Figure 10, the initial reaction rate shows a decrease when the water concentration is increased. 


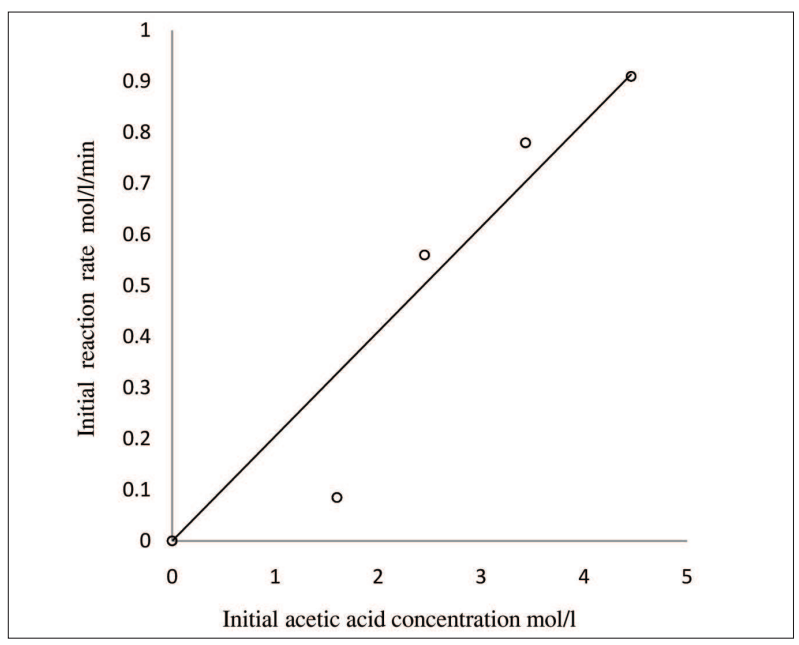

Figure 9: Effect of acid concentration on initial reaction rate at $335 \mathrm{~K}\left(5.4 \%(\mathrm{w} / \mathrm{w})\right.$ catalyst loading, $\mathrm{C}_{\mathrm{B}, 0}=1.6 \mathrm{~mol} \mathrm{~L}^{-1}$, $\left.\mathrm{C}_{\mathrm{E}, 0}=\mathrm{C}_{\mathrm{W}, 0}=0\right)$.

It can be predicted that there is an inhibiting effect on the reaction rate caused by the adsorption of water on the catalyst surface. Literature reveals that the inhibiting effect of ester on the reaction rate can be neglected (Altiokka \& Citak, 2003). The experimental results showing a strong adsorption of water and a negligible acid adsorption are in agreement with the assumptions that have been made in the reaction rate mechanism. A similar behaviour has been observed by Altiokka and Citak (2003), for the esterification of acetic acid with isobutanol over Amberlite IR-120 resin.

\section{CONCLUSION}

The esterification of acetic acid with ethyl alcohol over a cation exchange resin, Lewatit $\mathrm{S} 1467$ with $\mathrm{H}^{+}$ionic form was presented in this paper. The reaction rate was found to increase with the catalyst loading and temperature. The experimental kinetic data were correlated with both pseudo homogeneous and heterogeneous models, and the heterogeneous kinetic model correlated the kinetic data better than the pseudo homogeneous model. Heterogeneous models, ER and LH models both showed good agreement with the experimental kinetic data, although the LH model showed slightly better results. The activation energy was found to be about $58 \mathrm{kJmol}^{-1}$. The cation exchange resin, Lewatit $\mathrm{S} 1467$ in $\mathrm{H}^{+}$form showed a good catalytic performance in the esterification reaction of acetic acid with ethanol.

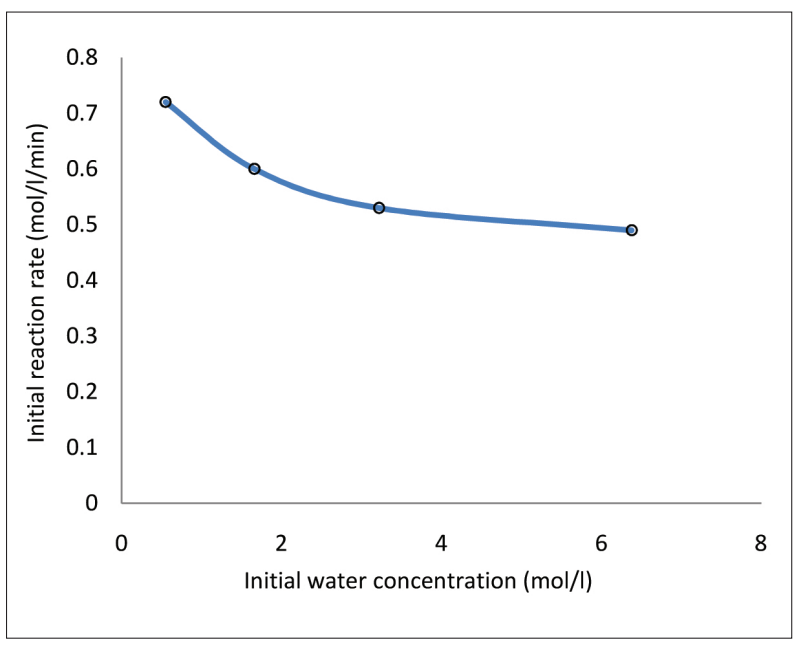

Figure 10: Effect of water concentration on initial reaction rate at $335 \mathrm{~K}\left(5.4 \%(\mathrm{w} / \mathrm{w})\right.$ catalyst loading, $\mathrm{C}_{\mathrm{A}, 0}=\mathrm{C}_{\mathrm{B}, 0}=1.6$ $\left.\mathrm{mol} \mathrm{L}^{-1}, \mathrm{C}_{\mathrm{E}, 0}=0\right)$.

\section{Acknowledgement}

This research was supported by the University of Moratuwa, Senate Research Grant Number SRC/ LT/2009/44.

\section{REFERENCES}

1. Akbay E.O. \& Altiokka M.R. (2011). Kinetics of esterification of acetic acid with n-amyl alcohol in the presence of amberlyst-36. Applied Catalysis A: General 396(1 - 2): $14-19$.

2. Alqahtani A., Teo H.T.R. \& Saha B. (2005). Esterification of dilute acetic acid with iso-amyl alcohol: heterogeneous kinetics and measurement of residue curve map. Proceedings of the $7^{\text {th }}$ World Congress of Chemical Engineering, Glasgow, Scotland, 10 - 14 July.

3. Altiokka M.R. \& Çitak A. (2003). Kinetics study of esterification of acetic acid with isobutanol in the presence of amberlite catalyst. Applied Catalysis A: General 239(1 - 2): 141 - 148 .

4. Carberry J. (1976). Chemical and Catalytic Reaction Engineering. McGraw-Hill, New York, USA.

5. Chakrabarti A. \& Sharma M.M. (1993). Cationic ion exchange resins as catalysts. Reactive Polymers 20(1-2): $1-45$.

6. Delgado P., Sanz M.T. \& Beltran S. (2007). Kinetic study for esterification of lactic acid with ethanol and hydrolysis of ethyl lactate using an ion exchange resin catalyst. Chemical Engineering Journal 126: 111 - 118. DOI: http://dx.doi.org/10.1016/j.cej.2006.09.004 
7. Erdem B. \& Cebe M. (2006). Kinetics of esterification of propionic acid with n-amyl alcohol in the presence of cation exchange resins. Korean Journal of Chemical Engineering 23(6): 896 - 901.

DOI: http://dx.doi.org/10.1007/s11814-006-0005-3

8. Fogler H.S. (2006). Elements of Chemical Reaction Engineering, $4^{\text {th }}$ edition. Prentice Hall, New Delhi, India.

9. Hangx G., Kwant G., Maessen H., Markusse P. \& Urseanu I. (2001). Reaction kinetics of the esterification of ethanol and acetic acid towards ethyl acetate. Technical Report. The Centre for Process Integration, University of Manchester. Available at http://www.cpi.umist.ac.uk/intint/NonConf_ Del/22.pdf, Accessed on 17 July 2014.

10. Helfferich F.G. (1962). Ion Exchange. MacGraw-Hill, New York, USA.

11. Izci A. \& Hosgun H.L. (2007). Kinetics of synthesis of isobutyl propionate over amberlyst-15. Turkish Journal of Chemistry 31(5): $493-499$.

12. Izci A., Uyar E. \& Izci E. (2009). Determination of adsorption and kinetic parameters for synthesis of isobutyl acetate catalyzed by Amberlite IR-122. Chemical Engineering Communications 196(1-2): 6-67.

13. Jagadeesh Babu P.E., Sandesh K. \& Saidutta M.B. (2011). Kinetics of esterification of acetic acid with methanol in the presence of ion exchange resin catalysts. Industrial and Engineering Chemistry Research 50(12): 7155 - 7160. DOI: http://dx.doi.org/10.1021/ie101755r

14. Ju I.B., Lim H.W., Jeon W., Suh D.J., Park M.J. \& Suh Y.W.H. (2011). Kinetic study of catalytic esterification of butyric acid and n-butanol over Dowex 50W $\times 8-400$. Chemical Engineering Journal 168(1): 293 - 302.

DOI: http://dx.doi.org/10.1016/j.cej.2010.12.086

15. Kirbaslar S.I., Baykal Z.B. \& Dramur U. (2001a). Esterification of acetic acid with ethanol catalyzed by an acidic ion-exchange resin. Turkish Journal of Chemistry 25: $569-577$.

16. Kirbaslar S.I., Terzioglu H.Z. \& Dramur U. (2001b). Catalytic esterification of methyl alcohol with acetic acid. Chinese Journal of Chemical Engineering 9(1): 90 - 96.

17. Lee M.J., Chiu J.Y. \& Lin H. (2002). Kinetics of catalytic esterification of propionic acid and n-butanol over amberlyst 35. Industrial and Engineering Chemistry Research 41(12): 2882 - 2887.

DOI: http://dx.doi.org/10.1021/ie0105472

18. Lee M.J., Wu H.T. \& Lin H. (2000). Kinetics of catalytic esterification of acetic acid and amyl alcohol over dowex. Industrial \& Engineering Chemistry Research 39(11): $4094-4099$.

DOI: http://dx.doi.org/10.1021/ie0000764

19. Lilja J., Aumo J., Salmi T., Murzin Y.D., Maki-Arvel P., Sundell M., Ekman K., Peltonen R. \& Vainio H. (2002). Kinetics of esterification of propionic acid with methanol over a fibrous polymer-supported sulphonic acid catalyst. Applied Catalysis A: General 228: 253 - 267.
20. Liu W.T. \& Tan C.S. (2001). Liquid-phase esterification of propionic acid with n-butanol. Industrial and Engineering Chemistry Research 40(15): 3281 - 3286.

DOI: http://dx.doi.org/10.1021/ie001059h

21. Liu Y., Lotero E. \& Goodwin Jr. J.G. (2006). A comparison of the esterification of acetic acid with methanol using heterogeneous versus homogeneous acid catalysis. Journal of Catalysis 242: 278 - 286.

DOI: http://dx.doi.org/10.1016/j.jcat.2006.05.026

22. Mazzotti M., Neri B., Gelosa D., Kruglov A. \& Morbidelli M. (1997). Kinetics of liquid-phase esterification catalyzed by acidic resin. Industrial and Engineering Chemistry Research 36(1): 3 - 10.

DOI: http://dx.doi.org/10.1021/ie960450t

23. Peters T.A., Benes N.E. \& Holmen A. (2006). Comparison of commercial solid acid catalysts for the esterification of acetic acid with butanol. Applied Catalysis A: General 297(2): $182-188$.

DOI: http://dx.doi.org/10.1016/j.apcata.2005.09.006

24. Reid R.C., Prausnitz J.M. \& Poling B.E. (1987). Properties of Gases and Liquids, $4^{\text {th }}$ edition. McGraw Hill, New York, USA.

25. Schwarzer R. (2006). Esterification of acetic acid with methanol: a kinetic study on amberlyst 15. MSc Thesis (Chemical Engineering), University of Pretoria. Available at: http://upetd.up.ac.za/thesis/submitted/etd-05152007112055/unrestricted/00dissertation.pdf, Accessed on 17 July 2014.

26. Shanmugam S., Viswanathan B. \& Varadarajan T.K. (2004). Esterification by solid acid catalysts: a comparison. Journal of Molecular Catalysis A: Chemical 223: 143 $-147$.

DOI: http://dx.doi.org/10.1016/j.molcata.2004.02.030

27. Smith J.M. (1970). Chemical Engineering Kinetics, $2^{\text {nd }}$ edition. McGraw Hill, New York, USA.

28. Toor A.P., Sharma M., Kumar G. \& Wanchoo R.K. (2011). Kinetic study of esterification of acetic acid with nbutanol and isobutanol catalyzed by ion exchange resin. Bulletin of Chemical Reaction Engineering and Catalysis 6(1): $23-30$.

DOI: http://dx.doi.org/10.9767/bcrec.6.1.665.23-30

29. Tsai Y.T., Lin H. \& Lee M.(2011). Kinetics of heterogeneous esterification of glutaric acid with methanol over amberlyst 35. Journal of the Thaiwan Institute of Chemical Engineers 42: $271-277$.

DOI: http://dx.doi.org/10.1016/j.jtice.2010.07.010

30. Yadav G.D. \& Thathagar M.B. (2002). Esterification of maleic acid with ethanol over cation exchange resin catalysts. Reactive Functional Polymers 52(1): 99 - 110.

31. Zhang Y., Ma L. \& Yang J. (2004). Kinetics of esterification of lactic acid with ethanol catalyzed by cation-exchange resins. Reactive Functional Polymers 61(1): 101 - 114. DOI: http://dx.doi.org/10.1016/j.reactfunctpolym.2004.04.003 